\title{
Bifurcation in the Steady-State Height of Colloidal Particles near an Electrode in Oscillatory Electric Fields: Evidence for a Tertiary Potential Minimum
}

\author{
T. J. Woehl, ${ }^{1, *}$ B. J. Chen, ${ }^{1}$ K. L. Heatley, ${ }^{1}$ N. H. Talken, ${ }^{1}$ S. C. Bukosky, ${ }^{1}$ C. S. Dutcher, ${ }^{1,2, \dagger}$ and W. D. Ristenpart ${ }^{1, \ddagger}$ \\ ${ }^{1}$ Department of Chemical Engineering and Materials Science, \\ University of California Davis, Davis, California 95616, USA \\ ${ }^{2}$ Air Quality Research Center, University of California Davis, Davis, California 95616, USA
}

(Received 5 August 2014; revised manuscript received 8 December 2014; published 27 February 2015)

Application of an oscillatory electric field is known to alter the separation distance between micron-scale colloidal particles and an adjacent electrode. This behavior is believed to be partially due to a lift force caused by electrohydrodynamic flow generated around each particle, with previous work focused on identifying a single steady-state "height" of the individual particles over the electrode. Here, we report the existence of a pronounced bifurcation in the particle height in response to low-frequency electric fields. Optical and confocal microscopy observations reveal that application of a $\sim 100 \mathrm{~Hz}$ field induces some of the particles to rapidly move several particle diameters up from the electrode, while the others move closer to the electrode. Statistics compiled from repeated trials demonstrate that the likelihood for a particle to move up follows a binomial distribution, indicating that the height bifurcation is random and does not result from membership in some distinct subpopulation of particles. The fraction of particles that move up increases with increased applied potential and decreased frequency, in a fashion qualitatively consistent with an energy landscape predicated on competition between electrohydrodynamic flow, colloidal interactions, and gravitational forces. Taken together, the results provide evidence for the existence of a deep tertiary minimum in the effective electrode-particle interaction potential at a surprisingly large distance from the electrode.

DOI: 10.1103/PhysRevX.5.011023

\section{INTRODUCTION}

Seminal work by Perrin on sedimentation profiles of colloidal particles in a gravitational field showed that the volume fraction of particles decays exponentially from the bottom surface, with the so-called gravitational length scale determined by a balance of thermal diffusive forces pushing the particles up and gravity pulling them down [1]. The Derjaguin-Landau-Verwey-Overbeek (DLVO) theory was later developed to include the effects of Coulombic forces and van der Waals attraction to model the behavior of charged colloidal particles in close proximity to a charged surface; integrating the sum of the DLVO and gravitational forces yields the well-known interaction potential that

\footnotetext{
*Present address: Applied Chemical and Materials Division, National Institute of Standards and Technology, Boulder, Colorado 80305, USA.

${ }^{\dagger}$ Present address: Department of Mechanical Engineering, University of Minnesota, Minnesota 55455, USA.

Corresponding author. wdristenpart@ucdavis.edu

Published by the American Physical Society under the terms of the Creative Commons Attribution 3.0 License. Further distribution of this work must maintain attribution to the author(s) and the published article's title, journal citation, and DOI.
}

Subject Areas: Condensed Matter Physics, Fluid Dynamics, Soft Matter contains the primary and secondary potential minima [2]. This classical picture is dramatically altered, however, by application of an external electric field. A variety of additional phenomena, including electrophoresis [3], dielectrophoresis [4,5], induced dipolar interactions [6,7], electro-osmotic flow [2], electrohydrodynamic (EHD) flow [8-10], and induced charge electro-osmosis (ICEO) [11-13], are known to significantly alter the particle behavior and give rise to new particle configurations. In particular, colloids have been widely observed to form planar aggregates near an electrode surface in response to an oscillatory electric field applied normal to the electrode [7-9,14-28]. Although there is broad agreement that electrically induced fluid flow pushes the particles to aggregate laterally, the relative contribution of different types of proposed flows-including EHD [10,21], Faradaically coupled electro-osmotic flow (FCEO) [22], and ICEO [22,26] - remains unclear. Two key aspects complicate theoretical interpretation. First, particles are observed to aggregate rapidly when suspended in certain electrolytes (e.g., $\mathrm{KCl}$ ), but rapidly separate in others (e.g., $\mathrm{KOH})[24,29]$. Despite much investigation, extant theoretical models $[26,27]$ are unable to predict particle behavior a priori for a given electrolyte. A second and related complicating factor is that the separation distance between the colloidal particles and the electrode, i.e., the 
particle "height," is believed to affect the net magnitude of electrically induced flows around the particles [10,21], but the force balance controlling the particle height itself depends on the magnitude of the flow [28]. Sides and co-workers have used total internal reflection microscopy to study the nanometer-scale oscillations in particle height, and have established that while electrophoresis is the predominant force causing oscillatory motion, there are hydrodynamic interactions that cause the particle oscillations to be nonsymmetric in time and out of phase with the electric field [30]. They further established that the phase angle between the applied field and the particle height was correlated with particle separation or aggregation, and that the time-average height varied with electrolyte type, frequency, and field strength [13,23,26,27]. More recently, Dutcher et al. used confocal microscopy to demonstrate that the average particle height is correlated with both the magnitude of the lateral flow as well as the effective particle diffusivity and consequent propensity to form disordered or hexatic aggregates [28]. Despite extensive investigation $[22,23,26,27,29]$, however, extant theoretical models are unable to predict height and corresponding particle behavior a priori for a given electrolyte.

In all previous work, the implicit assumption has been that there is a single steady-state height of the colloids over the electrode. Here, we report the existence of a striking bifurcation in the particle height, for particles immersed in electrolytes that yield lateral particle separation. Specifically, application of a low-frequency $(<300 \mathrm{~Hz})$ electric field causes micron-scale colloids resting near an electrode to migrate vertically to one of two widely separated steady-state heights above the electrode. Particles moving up migrate rapidly to a height several particle diameters above the electrode, while particles moving down migrate to a height on the order of $0.5 \mu \mathrm{m}$. Repeated trials establish that the probability for any given particle to move up follows a random binomial distribution, and that the fraction of particles that moves up depends sensitively on the particle type, suspending electrolyte, and electric field strength and frequency. We demonstrate that a force balance that includes typical DLVO forces, gravity, and a repulsive drag force due to EHD flow yields an interaction energy landscape with a tertiary minimum at a position from the electrode that is qualitatively consistent with the observed height bifurcation. Finally, we discuss the implications for understanding the underlying flow mechanisms giving rise to the lateral aggregation or separation behavior, as well as potential applications that can exploit a deep tertiary minimum at such large distances from the solid surface.

\section{COLLOIDAL BIFURCATION EXPERIMENTS}

Our experimental method is similar to previous work detailed elsewhere $[21,28,29]$. In short, the experimental apparatus consists of parallel glass slides coated with
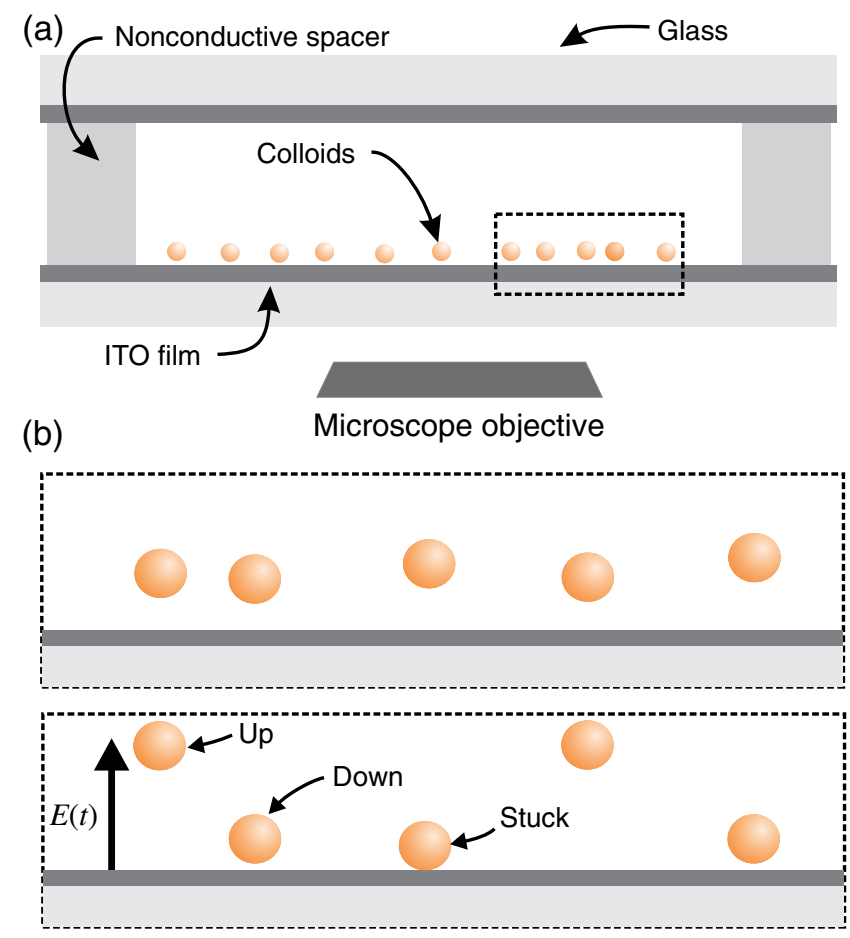

FIG. 1. (a) Schematic of the experimental apparatus (not to scale). (b) Schematic indicating particle heights before and after application of the electric field (top and bottom, respectively). Note that three possible heights occur: "up," "down," and "stuck" (i.e., irreversibly adhered to the electrode).

indium tin oxide (15 $\Omega$ sheet resistance) separated by a 1-mm-thick nonconductive polydimethylsiloxane (PDMS) spacer (Fig. 1). A number of different particles are tested: $2-\mu \mathrm{m}$-diameter fluorescent sulfated polystyrene (PS) (Invitrogen, USA), $2 \mu \mathrm{m}$ aliphatic amine PS (Invitrogen, USA), and $2 \mu \mathrm{m}$ sulfated PS (Invitrogen, USA). Aliphatic amine PS is suspended in $1 \mathrm{mM} \mathrm{HCl}$ (electrophoretic mobility $u_{e}=5.9 \times 10^{-8} \mathrm{~m}^{2} \mathrm{~s}^{-1} \mathrm{~V}^{-1}$, zeta potential $\zeta_{p}=$ $75 \mathrm{mV})$ and $\mathrm{HNO}_{3}\left(u_{e}=7.0 \times 10^{-8} \mathrm{~m}^{2} \mathrm{~s}^{-1} \mathrm{~V}^{-1}, \quad \zeta_{p}=\right.$ $88 \mathrm{mV}$ ), and the sulfate and fluorescent sulfated PS are suspended in $1 \mathrm{mM} \mathrm{NaOH}\left(u_{e}=-9.6 \times 10^{-8} \mathrm{~m}^{2} \mathrm{~s}^{-1} \mathrm{~V}^{-1}\right.$, $\zeta_{p}=-121 \mathrm{mV}$ and $u_{e}=-8.7 \times 10^{-8} \mathrm{~m}^{2} \mathrm{~s}^{-1} \mathrm{~V}^{-1}, \zeta_{p}=$ $-110 \mathrm{mV}$, respectively). The electrophoretic mobilities are measured using dynamic light scattering (Malvern Zetasizer), while the zeta potentials are ostensible (approximate) values calculated using the HelmholtzSmoluchowski relation for small electrophoretic mobilities [31]. Prior to each experiment, the particles are washed and diluted to $1 \times 10^{-6}$ volume fraction in the desired electrolyte. Particles are allowed to settle near the bottom electrode by gravity for $\sim 2 \mathrm{~h}$, after which they are observed to move laterally by Brownian motion, indicating that they remain separated from the electrode by colloidal scale forces. The height bifurcations are observed with either a transmission optical microscope or a laser scanning confocal microscope (Olympus FV1000). For the confocal microscopy, a $5 \mu \mathrm{L}$ drop of the suspension is first placed on 
the bottom electrode and allowed to dry before completely filling the cell. This allows a small number of particles to adhere irreversibly to the electrode, providing a reference for the direct height measurements (see Ref. [28] and Supplemental Material [32] for details of the confocal microscopy technique). A function generator is used to apply voltages ranging from 0.5 to $10 \mathrm{~V}$ peak to peak (Vpp) and $f=50-500 \mathrm{~Hz}$.

\section{RESULTS AND DISCUSSION}

\section{A. Direct height measurements}

Figure 2 shows representative false-colored confocal microscope images of approximately 10 individual particles $(2 \mu \mathrm{m}$ sulfated PS particles in $1 \mathrm{mM} \mathrm{NaOH})$ before
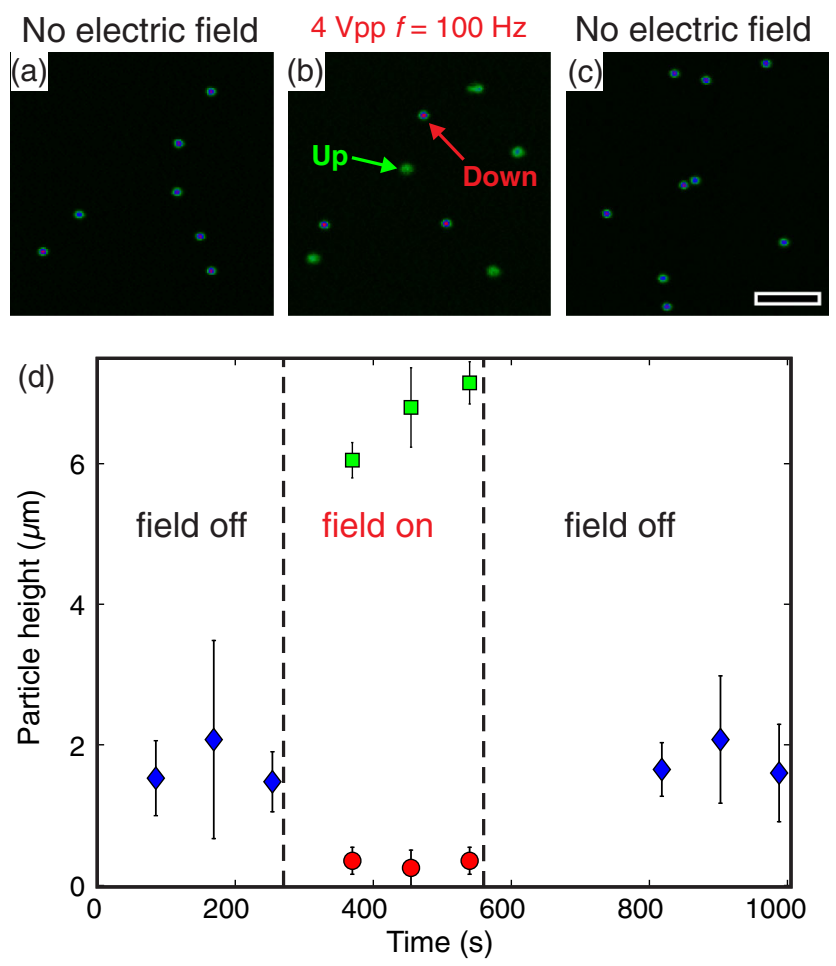

FIG. 2. (a)-(c) Representative false-colored confocal microscopy images of the height bifurcation exhibited by fluorescent $2 \mu \mathrm{m}$ sulfated PS particles in $1 \mathrm{mM} \mathrm{NaOH}$. (a) Prior to application of the field, the particles are all in the same focal plane adjacent to the electrode. (b) $30 \mathrm{~s}$ after application of a $4 \mathrm{Vpp}, 100 \mathrm{~Hz}$ field, the particles have moved to two distinct focal planes. Particles with red centers are approximately in focus, bright green particles are several microns above focus. (c) $5 \mathrm{~min}$ after the field is removed, the particles have moved back to their original focal plane. The scale bar in (c) is $10 \mu \mathrm{m}$. (d) Electrode-to-surface particle heights extracted from confocal $z$ scans. Vertical dashed lines indicate when the electric field is applied and removed. For the "field off" conditions, the heights are the mean of eight particle heights. For the "field on" condition, the heights are the mean of four particle heights in each widely separated focal plane. Error bars are 2 standard deviations around the mean. and after application of the electric field. With no electric field applied, the particles are mobile and all have similar fluorescence intensities, indicating that the particles are at approximately the same height above the electrode [Fig. 2(a)]. The particle intensities fluctuate slightly but randomly with time, indicating that the particles are also moving normal to the electrode surface via Brownian motion. When an electric field is applied, approximately half of the particles rapidly move out of the focal plane within just a few seconds, which is manifested as a large change in their fluorescence intensity [bright green particles, Fig. 2(b)]. The rest of the particle intensities do not change noticeably by eye; they have intensities similar to that of the particles stuck to the electrode, indicating that they are within several hundred nanometers of the electrode surface (cf. Movie 1 in Supplemental Material [32]). The particle height bifurcation is observed to be stable over time scales of tens of minutes; in other words, the "up" and "down" particles remain at their respective heights indefinitely, albeit with minor intensity fluctuations consistent with Brownian motion. Furthermore, after the initial transient, no particles are observed to switch from one focal plane to another. When the electric field is removed, the particles typically settle back to the same focal plane within 5 min of removing the field [Fig. 2(c)].

The particle heights relative to the electrode surface are directly measured using confocal microscopy; refer to the Supplemental Material for details on the confocal microscopy method (Fig. S1) [32]. Figure 2(d) shows the absolute heights of particles before, during, and after an oscillatory electric field is applied. Three consecutive 85-s-long $z$ scans of the particles are acquired for each condition, where each $z$ scan yields a single particle height [time points in Fig. 2(d) correspond to the time at the end of each $z$ scan]. With no electric field applied, all of the particles are $1-3 \mu \mathrm{m}$ away from the electrode surface $(t=0-250 \mathrm{~s}$, blue diamonds). At $t=270 \mathrm{~s}$, the electric field is applied, the particle heights are allowed to stabilize at their respective heights for $15 \mathrm{~s}$, and three more consecutive $z$ scans are obtained. The particles whose fluorescence intensities dramatically change when the field is applied [cf. bright green particles, Fig. 2(b)] have moved up approximately $5 \mu \mathrm{m}$ from their previous equilibrium position [green squares, Fig. 2(d)]. While a fluorescent intensity change is not observed by eye for the particles that stay down, the height measurements reveal that they in fact have moved slightly closer toward the electrode to a height of approximately $0.5 \mu \mathrm{m}$ [red circles, Fig. 2(d)]. While particle movement toward the electrode in an oscillatory electric field is consistent with previous observations in a similar system $[13,30,33]$, movement of particles to two distinct steady-state heights, one of which is several particle diameters away from the electrode, has not been previously reported. Notably, the error bars in the particle heights for both the up and down particles decrease compared to those 
observed in the absence of the field, suggesting that both populations of particles occupy a deeper effective potential energy well than when there is no electric field. At $550 \mathrm{~s}$, the electric field is removed and the particles are allowed to settle back to their equilibrium positions for $180 \mathrm{~s}$. Three more consecutive $z$ scans are then obtained [blue diamonds, Fig. 2(d)], showing that the particles have indeed moved back to approximately the same average equilibrium height. The height bifurcation is reproducible over several trials without any observable hysteresis effects in the particle heights (data not shown), provided the electric field magnitude is the same in each trial.

\section{B. Colloidal height-bifurcation probability}

One possible explanation for the height bifurcation is that the colloidal suspension contains a distinct subpopulation of particles with different properties (e.g., zeta potential) that causes them to always move up. Our qualitative observations, however, suggest that the likelihood for a single particle to move up is random since individual particles are observed to move either up or down, seemingly at random, over numerous repeated height

(a)
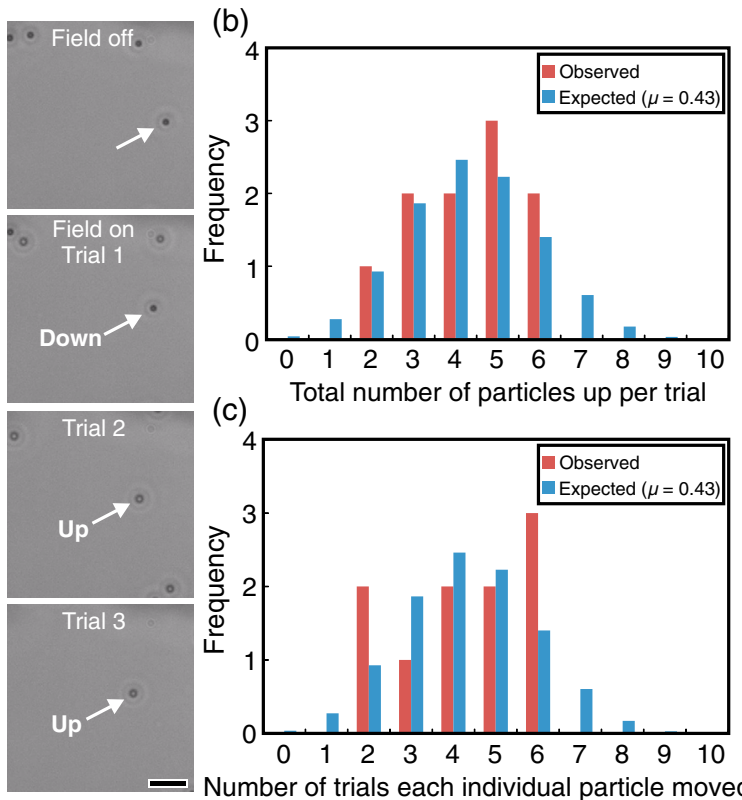

(c)

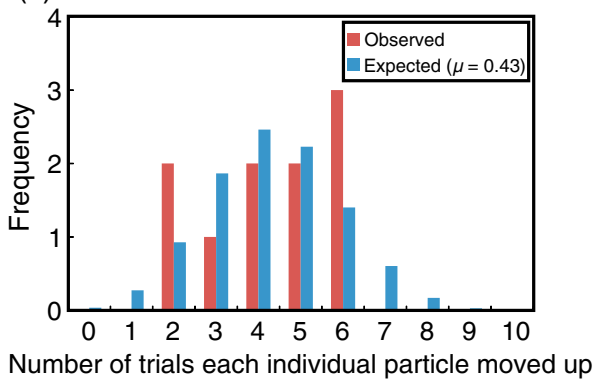

FIG. 3. Binomial statistics for the 10 bifurcation trials represented in Table I. Statistics were acquired for $2 \mu \mathrm{m}$ sulfated polystyrene suspended in $1 \mathrm{mM} \mathrm{NaOH}$ with an applied potential of $5 \mathrm{Vpp}, 100 \mathrm{~Hz}$. (a) Representative images of particle bifurcation trials in the optical microscope. The white arrows follow the same particle (particle 9 in Table I) through each bifurcation trial, indicating whether the particle moved up or down when the potential was applied. (b) Histogram of the total number of particles that moved up per trial. (c) Histogram of the number of trials where an individual particle moved up. Both histograms are compared to a binomial distribution with an expected probability of success of $\mu=0.43$, which is the average probability for the particles to move up over 10 trials. bifurcations. To quantitatively test whether the height bifurcation is random, we perform 10 consecutive bifurcation trials at $5 \mathrm{Vpp}, 100 \mathrm{~Hz}$ and observe the behavior of 50-75 particles in an optical microscope. The optical microscope does not yield absolute height information, but the dramatic change in height and focal plane of the particles allows a convenient way to quantify the fraction of particles that move up. We randomly choose 10 particles in the field of view and note whether each individual particle moves up or down during each trial. In the optical microscope, height change is identified by a marked defocus in the particle [cf. Fig. 3(a), compare no field, trial 1 (down), and trials 2 and 3 (up)]. Table I summarizes the results of the 10 trials, in which moving up is denoted with a "u" and moving down is denoted with a "d." The table clearly shows that the same particle readily occupies either focal plane; for example, particle no. 4 goes up on the first two trials, then down on the third trial, then up again on the fourth trial, and so on.

The overall observed probability for a particle to move up (in this specific case) is $\mu=0.43 \pm 0.16$, where the indicated error is 2 standard deviations of the mean. To test whether the particle bifurcation is random, we compare the probability data from the 10 trials to a binomial distribution with an expected probability of success of $\mu=0.43$. We test two distinct null hypotheses: (1) there is no statistically significant difference from a binomial distribution for how many particles moved up per trial, and (2) there is no statistically significant difference from a binomial distribution for how many times an individual particle moves up. In simple terms the first hypothesis addresses the question, for 10 trials, did a statistically unlikely total number of particles move up? Likewise, the second hypothesis addresses the question, did any of the 10 particles tested move up a statistically unlikely number of times?

TABLE I. Consecutive height bifurcation trials of 10 randomly chosen sulfated polystyrene particles in $1 \mathrm{mM} \mathrm{NaOH}$ with a $5 \mathrm{Vpp}, 100 \mathrm{~Hz}$ applied potential. The rows correspond to each trial while the columns correspond to each specific individual particle; the same individual particles are tracked through all 10 trials. A "d" indicates a particle that moves down, and a " $u$ " indicates a particle that moves up [cf. Figs. 1(b) and 2(b)].

\begin{tabular}{cccccccccccc}
\hline \hline & \multicolumn{10}{c}{ Particle } \\
\hline Trial & & 1 & 2 & 3 & 4 & 5 & 6 & 7 & 8 & 9 & 10 \\
& 1 & d & d & d & u & u & d & d & d & d & d \\
& 2 & d & u & d & u & d & d & d & d & u & d \\
& 3 & d & u & u & d & u & d & d & u & u & u \\
& 4 & u & d & d & u & d & u & u & d & u & u \\
& 5 & d & d & u & d & u & u & d & u & u & d \\
& 6 & d & u & d & d & d & u & u & u & d & d \\
& 7 & d & u & d & d & u & d & u & u & d & d \\
& 8 & u & u & d & u & d & u & d & d & u & d \\
& 9 & d & d & d & u & d & d & u & d & u & d \\
& 10 & u & u & d & u & d & d & u & u & d & d \\
\hline \hline
\end{tabular}


Figure 3(b) shows a histogram of the total number out of 10 particles that go up during each trial (red bars), compared to the expected number for a binomial distribution (blue bars). The distribution is clearly centered around 4-5 particles; i.e., during any given trial, approximately half of the particles move up. Figure 3(c) shows a histogram of the number of times each individual particle moves up, again compared to the expected number for a binomial distribution. Importantly, we see that none of the particles exhibit a preference for always (or never) moving up: the distribution is again centered around 4-5. To test the two null hypotheses, we compare the $\chi^{2}$ values obtained from comparing the experimental and binomial distributions for the number of particles up per trial and the number of times an individual particle moves up. Recall that $\chi^{2}$ is the sum of the squared difference between the expected and observed experimental values, and the value of $\chi^{2}$ is associated with a $p$ value, indicating statistical significance. The $p$ value for the number of particles up per trial [Fig. 3(b)] is $p=0.998$, indicating that we cannot reject null hypothesis (1). Similarly, the $p$ value for the number of times each individual particle moves up [Fig. 3(c)] is $p=0.911$, thus also failing to reject null hypothesis (2). We conclude that the likelihood for a particle to move up during the height bifurcation is random, rather than resulting from membership in some distinct subpopulation of particles.

\section{Electric field dependence}

The results described above focus on $2 \mu \mathrm{m}$ PS particles immersed in $1 \mathrm{mM} \mathrm{NaOH}$. Additional experiments with various particle types, particle sizes, and suspending electrolytes confirm that the height bifurcation is a robust and reproducible phenomenon. We observe height bifurcations for a number of particle-electrolyte combinations, including $2 \mu \mathrm{m}$ sulfated PS and fluorescent sulfated PS in $1 \mathrm{mM} \mathrm{NaOH}$ (cf. Figs. 2 and 3 ) and $2 \mu \mathrm{m}$ aliphatic amine $\mathrm{PS}$ in $1 \mathrm{mM} \mathrm{HCl}$ and $\mathrm{HNO}_{3}$ (not shown). Likewise, the height bifurcation is observed on both ITO electrodes and platinum electrodes. The particle bifurcation is qualitatively similar for each particle-electrolyte combination, although the fraction of particles that move up is dependent on the electric field parameters, particle type, and background electrolyte. Notably, however, we never observe any height bifurcation for particles suspended in electrolytes that induce lateral aggregation (e.g., $\mathrm{KCl}, \mathrm{NaCl}$ ).

To systematically test the electric field dependence of the particle bifurcation, we perform systematic heightbifurcation trials with $2 \mu \mathrm{m}$ sulfated PS in $1 \mathrm{mM} \mathrm{NaOH}$ and $2 \mu \mathrm{m}$ aliphatic amine PS in $1 \mathrm{mM} \mathrm{HCl}$ (Fig. 4), again using optical microscopy. To minimize the possibility of any systematic errors, the varied voltage and frequency experiments are performed in random order. The fraction of particles that move up in $1 \mathrm{mM} \mathrm{NaOH}$ at a fixed frequency of $100 \mathrm{~Hz}$ [Fig. 4(a), green triangles] increases
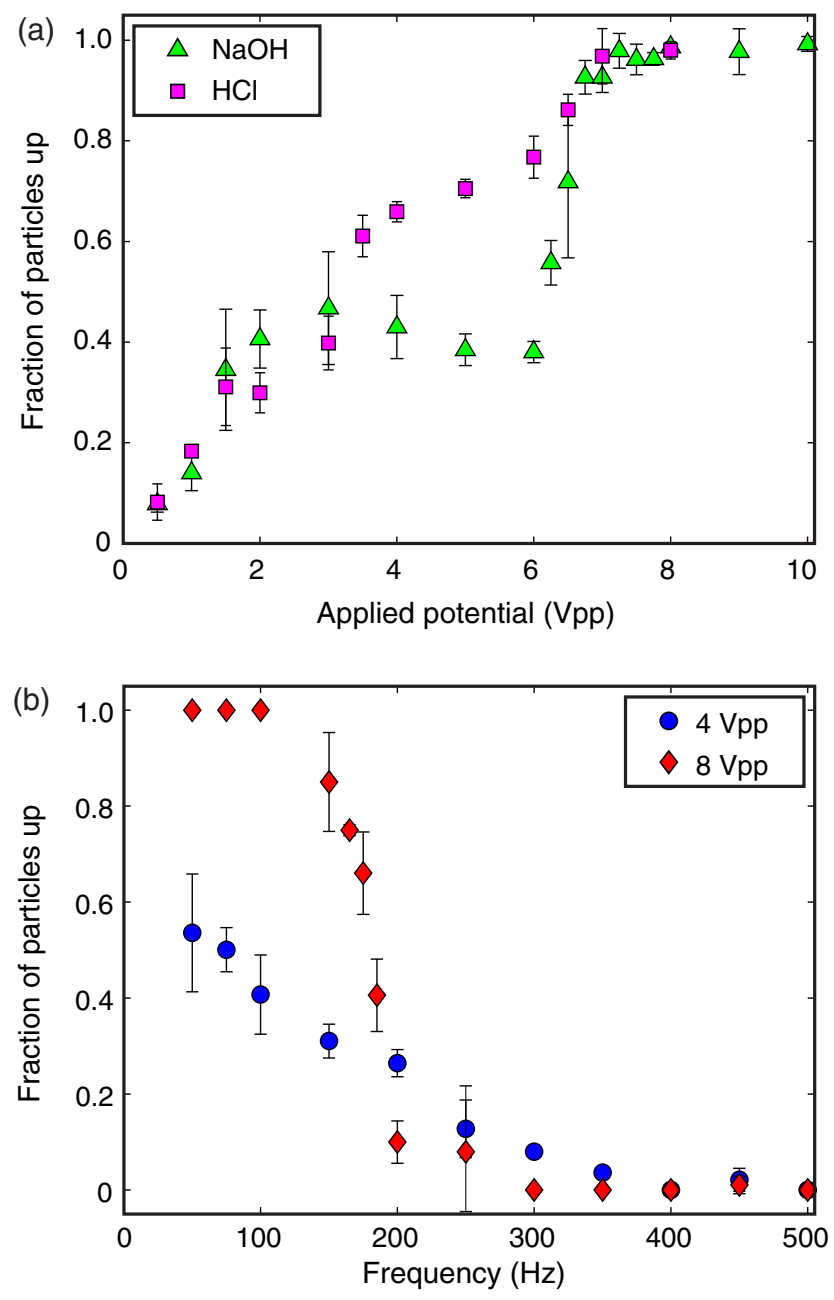

FIG. 4. Electric field dependence of the particle height bifurcation. (a) Fraction of particles that move up as a function of applied potential for $2 \mu \mathrm{m}$ sulfated PS in $1 \mathrm{mM} \mathrm{NaOH}$ (green triangles) and $2 \mu \mathrm{m}$ aliphatic amine PS in $1 \mathrm{mM} \mathrm{HCl}$ (magenta squares). The frequency is held constant at $100 \mathrm{~Hz}$. (b) Fraction of $2 \mu \mathrm{m}$ sulfated PS particles that move up in $1 \mathrm{mM} \mathrm{NaOH}$ as a function of frequency for two applied potentials indicated in the figure. Each data point is the mean of at least three trials, error bars are 2 standard deviations of the mean.

monotonically from 0.05 to 0.45 between amplitudes of 0.5-3 Vpp, and then plateaus near 0.4 from 3-6 Vpp. Above $6 \mathrm{Vpp}$, there is a sharp increase in the fraction of particles that move up, reminiscent of a second-order phase transition: in this case, a transition from a bifurcated state to an unbifurcated state with all the particles moving up. The aliphatic amine functionalized particles in $1 \mathrm{mM} \mathrm{HCl}$ behave similarly to the sulfated PS at applied potentials $<3 \mathrm{Vpp}$; however, their behavior differs above $3 \mathrm{Vpp}$ [Fig. 4(a), magenta squares]. The fraction of particles that move up increases monotonically from 0.5 to $7 \mathrm{Vpp}$, with a smooth transition between the bifurcated and unbifurcated phase states. The difference in behavior between the aliphatic amine and sulfate functionalized PS might be 
due to different surface functionalities and opposite surface charges; the amine PS in $1 \mathrm{mM} \mathrm{HCl}$ has a zeta potential of $+75 \mathrm{mV}$ while the sulfated PS in $1 \mathrm{mM} \mathrm{NaOH}$ has a zeta potential of $-121 \mathrm{mV}$. In both cases, however, effectively all of the particles move up to the higher height for voltages greater than $7 \mathrm{Vpp}$.

Systematic tests of the effect of frequency reveal that higher frequencies decrease the propensity for particles to move up [Fig. 4(b)]. At 4 Vpp, sulfated PS particles in $1 \mathrm{mM} \mathrm{NaOH}$ are height bifurcated from 50 to $400 \mathrm{~Hz}$; the fraction of particles that move up decreases monotonically from about 0.5 at $50 \mathrm{~Hz}$ to 0 at $400 \mathrm{~Hz}$. At $8 \mathrm{Vpp}, 100 \%$ of the particles move up between $50-100 \mathrm{~Hz}$ [consistent with Fig. 4(a)], but undergo a sharp transition to height bifurcations in the frequency range of 150 to $300 \mathrm{~Hz}$. Above $300 \mathrm{~Hz}$ (at $8 \mathrm{Vpp}$ ), effectively none of the particles move up.

\section{Scaling analysis for a tertiary minimum}

Why does the particle height bifurcate? A crucial aspect of the observed behavior is that there are three distinct steady-state heights at which the particles may reside: "stuck" to the electrode, "down" near the electrode, and "up" far from the electrode [cf. Fig. 1(b)]. Importantly, the particles reside at each height indefinitely (while the field is applied) without switching to other heights, suggesting that each distinct height is defined by an energy minimum with a depth that is large compared to the thermal energy, $k_{B} T$. If the stuck height represents the classical primary minimum in the interaction energy, and the down height represents the influence of a secondary minimum, then the up height must represent the influence of a strong "tertiary" minimum. The electric field dependence shown in Fig. 4 suggests that increasing the applied voltage or decreasing the frequency increases the favorability for particles to move up to the ternary steady-state position.

Possible physical mechanisms for the observed behavior include FCEO [13], electrothermal flows [34], dielectrophoresis (DEP) [5], and EHD flow [10,21]. FCEO was originally proposed by Fagan et al. to explain aggregation or separation of particles by acting in concert with an $O(E)$ electrokinetic flow on the particle surface [22]. The weak $O\left(E^{2}\right)$ FCEO flow was proposed to break the symmetry of the electrokinetic flow, effecting either aggregation or separation of particles depending on the suspending electrolyte. In the context of the particle height bifurcation, the only capacity in which FCEO flow could contribute is by creating a lift force on the particles. However, Fagan et al. noted that the FCEO flow is too weak to induce aggregation or separation on its own $[13,22]$, so we conclude that any lift force on the particle produced by FCEO is too weak to contribute significantly to the height bifurcation. Likewise, electrothermal flows do not likely contribute to the particle height bifurcation, since the calculated amount of Joule heating due to the applied ac potential is negligible [28]. DEP at first seems to be an attractive explanation because the DEP force induced by the electric field gradient normal to the electrode, which scales as $F_{\text {DEP }} \sim \nabla|E|^{2}$, should be repulsive for the particles in our experiment [5]. This is seemingly in line with our experimental observations of some particles moving up during the height bifurcation; however, due to the short Debye length of our electrolytes $\left(\kappa^{-1} \approx 10 \mathrm{~nm}\right)$, the electric field enhancement and associated field gradient normal to the electrode decays to zero at distances $>\sim 50 \mathrm{~nm}$ from the electrode surface. In other words, the DEP force is vanishingly small at the large micron-scale distances associated with the particle height bifurcation.

Instead, a scaling analysis predicated on EHD flow appears to be broadly consistent with the experimental observations. Notably, the trend in the electric-fielddependent particle height bifurcation is qualitatively similar to previous experimental measurements of the lateral component of the EHD fluid flow generated around colloids near electrodes: the flow is observed to scale roughly as the square of the applied field and inversely with frequency; i.e., $U_{\mathrm{EHD}} \sim\left(E^{2} / \omega\right)$ [21]. The EHD flow scaling is consistent with systematic aggregation experiments using $2.7 \mu \mathrm{m}$ polystyrene particles suspended in $1 \mathrm{mM} \mathrm{KCl}$ [21]. Therefore, we hypothesize that an inward EHD fluid flow generated by each particle creates a lift force $[10,13]$, causing some particles to move into the tertiary minimum, while the rest of the particles are captured by the secondary minimum $[6,22,24,26,29]$. We model the bifurcation in particle height using a vertical force balance on the particle, consisting of short-ranged colloidal scale forces, such as double layer repulsion, van der Waals attraction, and gravity, as well as the more longranged repulsive EHD drag force. Specifically, the interaction pseudopotential energy is assumed to be the sum of

$$
\varphi_{\mathrm{total}}(h)=\varphi_{g}+\varphi_{\mathrm{vdW}}+\varphi_{\mathrm{dl}}+\varphi_{\mathrm{EHD}}
$$

where $\varphi_{g}$ is the gravitational potential, $\varphi_{\mathrm{vdW}}$ is the van der Waals potential, $\varphi_{\mathrm{dl}}$ is the interaction potential for double layer repulsion, and $\varphi_{\mathrm{EHD}}$ is the pseudopotential due to the EHD fluid drag in the vertical direction. The first three potential energies (gravity, van der Waals, and double layer) are readily estimated using classical expressions; see the Supplemental Material for details [32]. We also consider the contribution of induced dipole-dipole interactions, i.e., the attraction of the polarized particle to its image in the electrode [6], but omit it from the total interaction potential because it is negligible compared to the other interaction potentials (cf. Supplemental Material [32]). The new contribution here is the influence of the EHD fluid drag. Although fluid drag forces normally do not give rise to an interaction potential, here, the magnitude of the net flow depends sensitively on the particle height [21]. Accordingly, we refer to the EHD interaction potential as a 
"pseudopotential" because it is the result of a steady-state but nonequilibrium process.

To estimate the drag pseudopotential, we employ a previously derived asymptotic scaling analysis that treats the particle as a point dipole and calculates the electric stress induced by the tangential component of the particle dipole field on the surface charge of the electrode [21]. Although recent measurements suggest that the pointdipole EHD model fails to describe the observed dependence on the particle zeta potential [29], the model does capture the experimentally observed dependence on the square of the applied field strength $[21,28,29]$ and inverse of the frequency $[21,28]$. We invoke this model to obtain estimates of the fluid flow magnitude as a function of particle height over the electrode. Details on the derivation of the EHD drag pseudopotential are given in the Supplemental Material; here, we note that the interaction pseudopotential is

$\varphi_{\mathrm{EHD}}(h)=-6 \pi a^{4} b \varepsilon \varepsilon_{0} \kappa^{-1} E_{0}^{2}\left\{C_{0}^{\prime}+\frac{D \kappa^{2}}{\omega} C_{0}^{\prime \prime}\right\}\left(\frac{1}{b^{2}+h^{2}}\right)^{3 / 2}$,

where $E_{0}$ is the electric field strength, $\varepsilon_{0}$ is the vacuum permittivity, $\varepsilon$ is the dielectric constant of water, $C_{0}^{\prime}$ and $C_{0}^{\prime \prime}$ are the real and imaginary components of the particle dipole coefficient, $a$ is the particle radius, $D$ is the diffusion coefficient of the electrolyte, $\kappa^{-1}$ is the Debye length, $\omega$ is the angular frequency of the applied field, $h$ is the height of the particle over the electrode, and $b$ is the lateral distance from the particle center line to the position where the EHD flow is evaluated (cf. Fig. S3 in the Supplemental Material for a definition sketch [32]). For an EHD flow directed radially inward along the electrode towards the particle, the sign of $U_{\mathrm{EHD}}$ will be negative (because $\left\{C_{0}^{\prime}+\right.$ $\left.\left.\left(D \kappa^{2} / \omega\right) C_{0}^{\prime \prime}\right\}<0\right)$ and will generate a lift force on the particle. Note that the EHD interaction pseudopotential decays with increasing frequency and decreasing electric field. To model our experimental system, we use the parameter $b$, i.e., the lateral distance from the particle where the electrical stress is evaluated (cf. Fig. S3 [32]), as an effective fitting parameter. In this case, a value of $b=2 \mu \mathrm{m}$ yields a tertiary minimum at a height above the electrode qualitatively similar to experiments. Since $b$ is the same order of magnitude as the particle radius, $a=1 \mu \mathrm{m}$, the EHD drag pseudopotential scales approximately as $\varphi_{\mathrm{EHD}} \sim\left[1 /\left(1+h^{3}\right)\right]$. Thus, the tertiary minimum is formed by a balance of $\varphi_{\mathrm{EHD}}$ with the long-ranged gravitational potential that scales as $\varphi_{g} \sim h$.

We use the model described above with the relevant parameters for a $2 \mu \mathrm{m}$ sulfated PS particle in $1 \mathrm{mM}$ $\mathrm{NaOH}$ (cf. Table S1 [32]) to calculate the interaction potential between the particle and electrode (Fig. 5); individual potential curves for each interaction are shown in Fig. 5(a). Note that the two short-ranged DLVO
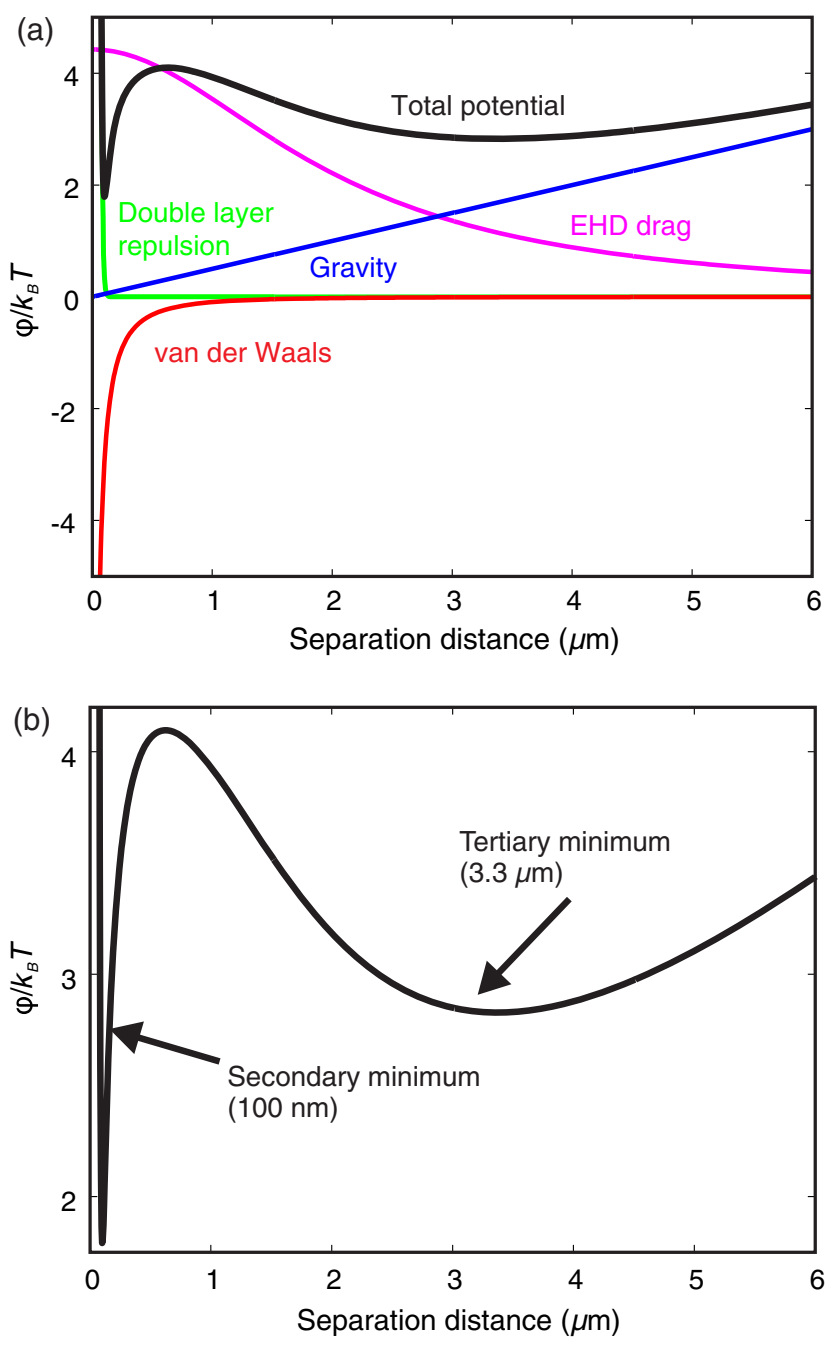

FIG. 5. Particle-electrode interaction potential for a $2 \mu \mathrm{m}$ sulfated PS particle in $1 \mathrm{mM} \mathrm{NaOH}$ with a $4 \mathrm{Vpp}, 100 \mathrm{~Hz}$ electric field applied. (a) Interaction potential profiles for each particle-electrode interaction included in the total potential [cf. Eqs. (1) and (2) herein and Eqs. (S1)-(S8) of Ref. [32]). (b) The total interaction potential [Eq. (1)]. Each potential is scaled to the thermal potential $\left(k_{B} T\right)$.

interactions, double layer repulsion (green curve) and van der Waals attraction (red curve), balance to form the well-known secondary minimum in the total interaction potential (black curve) [2]. The two long-range interactions, gravity (blue curve) and EHD lift (magenta curve), then form the tertiary minimum located at $3.3 \mu \mathrm{m}$.

There are several important features of the predicted interaction pseudopotential in Fig. 5(b) to note. First, the separation distances of the predicted secondary and tertiary potential minima show order of magnitude agreement with the direct height measurements (cf. Fig. 2). The "down" particles are measured to have a height of $\sim 500 \mathrm{~nm}$, qualitatively consistent with the value of $100 \mathrm{~nm}$ predicted by the vertical force balance [Fig. 5(b)]. The "up" particles have measured heights between $6-7 \mu \mathrm{m}$, within $50 \%$ of the 
predicted tertiary minimum position at $3.3 \mu \mathrm{m}$. Second, the shape of the pseudopotential minima accords with the variability observed in the particle heights measured using confocal microscopy. Relatively small error bars are observed for the down particle heights [cf. red circles, Fig. 2(d)], consistent with the calculated deep narrow secondary minima. Likewise, the comparatively larger error bars of the up particle heights [cf. green squares, Fig. 2(d)] suggest that these particles occupy a shallower potential well, which allows the particle height to fluctuate via Brownian motion. Again, this trend is captured well by the wider and shallower tertiary pseudopotential predicted by the scaling model [Fig. 5(b)]. Note that the model is based on a point-dipole scaling analysis of the EHD flow that ignores higher-order interactions between the particle and electrode. This may explain the quantitative discrepancies between the predicted position of the secondary and tertiary minima and the experimentally measured particle heights.

We likewise interpret the random nature of the particle height bifurcation (cf. Fig. 3) in terms of the interaction pseudopotential as follows. The measured equilibrium particle height with no electric field is highly variable compared to the height-bifurcated particles, with heights measured between $0.5-1.5 \mu \mathrm{m}$ [cf. Fig. 2(d), blue diamonds]. This observation suggests that the particles are located in a shallow potential well with no electric field applied, where the thermal potential of the particles is similar to that of the potential well depth. Consequently, we suggest that whether a particle moves up or down during the height bifurcation depends on its instantaneous height when the electric field is applied; i.e., particles closer to the tertiary minimum will "fall" into that potential well and move up and vice versa for particles proximate to the secondary minimum. Because the secondary and tertiary minima reside on either side of the equilibrium particle height with no electric field applied (cf. Fig. 2), a particle has the potential to end up in either minima when the electric field is applied. In other words, although whether or not a given particle moves up or down is random, one could presumably predict which direction it will move if the initial height of the particle at the instant the electric field is applied is known.

As a corollary, we hypothesize that the likelihood for a particle to move up depends on the depth of the tertiary minimum relative to the secondary; i.e., a deeper tertiary minimum should encourage a larger fraction of particles to move up during the bifurcation. The voltage- and frequency-dependent interaction potentials reveal trends that are qualitatively consistent with this hypothesis (Fig. 6). According to the interaction pseudopotential model, increasing the voltage increases the depth of the tertiary minimum relative to the secondary [Fig. 6(a)], in accord with an increasing number of particles moving up with increased voltage [Fig. 4(a)]. Alternatively, increasing frequency decreases the depth of the tertiary minimum relative to the secondary [Fig. 6(b)], again consistent with the decreasing number of particles moving up with increased frequency [Fig. 4(b)].

The separation distance and pseudopotential well depths of the secondary and tertiary minima show distinct regimes consistent with the electric-field-dependent particle heightbifurcation experiments (cf. Fig. 4). The potential well depth is taken as the difference between the minimum energy in each potential well and the inflection point between the two minima [Figs. 6(c) and 6(d)]. In the scaling model, the depth and position of the tertiary minimum is determined by the strength of the EHD lift force relative to gravity - the tertiary potential well depth therefore increases with increasing applied voltage and decreasing frequency. At applied voltages of 1-3 Vpp and a constant frequency of $100 \mathrm{~Hz}$ [conditions similar to Fig. 4(a)], the secondary minimum is predicted to be more than an order of magnitude deeper than the tertiary minimum [Fig. 6(c)]. Consistent with the potential well depths, the fraction of particles that move up during the bifurcation is relatively low in this voltage range [cf. Fig. 4(a)], indicating that the secondary minimum is dominating and capturing most of the particles. As the voltage is increased, the depth of the tertiary minimum increases (due to the EHD interaction pseudopotential scaling as $\varphi_{\mathrm{EHD}} \sim E_{0}^{2}$, while that of the secondary remains relatively unchanged, leading to an increase in the fraction of particles moving up above $3 \mathrm{Vpp}$ [cf. Fig. 4(a)]. For $1 \mathrm{mM} \mathrm{NaOH}$, the fraction of particles that move up is constant from 3-6 Vpp, while for $1 \mathrm{mM} \mathrm{HCl}$, the fraction increases monotonically [cf. Fig. 4(a)]. The behavior of the particles in $1 \mathrm{mM} \mathrm{HCl}$ is well described by the scaling model-further increase of the voltage causes the tertiary minimum to deepen further compared to the secondary minimum. However, the observed plateau in the fraction of up particles in $1 \mathrm{mM} \mathrm{NaOH}$ is inconsistent with the predicted interaction pseudopotentials. Further modeling and experiments will have to be performed to understand this feature. At a critical applied voltage of $\sim 7 \mathrm{Vpp}$, all of the particles move up, which is quantitatively consistent with the predicted dominant tertiary minimum at applied potentials $>7 \mathrm{Vpp}$ [Fig. 6(c)].

When increasing frequency from $50-350 \mathrm{~Hz}$ and holding voltage constant at $4 \mathrm{Vpp}$ [conditions similar to Fig. 4(b)], the depth of the tertiary minimum decreases due to the EHD interaction pseudopotential scaling as $\varphi_{\mathrm{EHD}} \sim 1 / \omega$ [Fig. 6(d)]. In this regime, neither minimum dominates, and the depth of the tertiary minimum decreases monotonically, which is qualitatively consistent with the gradual decrease in the fraction of particles that move up when increasing frequency [cf. blue circles Fig 4(b)]. Above $\sim 300 \mathrm{~Hz}$, no bifurcation is observed experimentally, consistent with the dominating secondary potential minima above $350 \mathrm{~Hz}$. While the electric-field-dependent potential minima predicted by the scaling analysis do not always quantitatively 

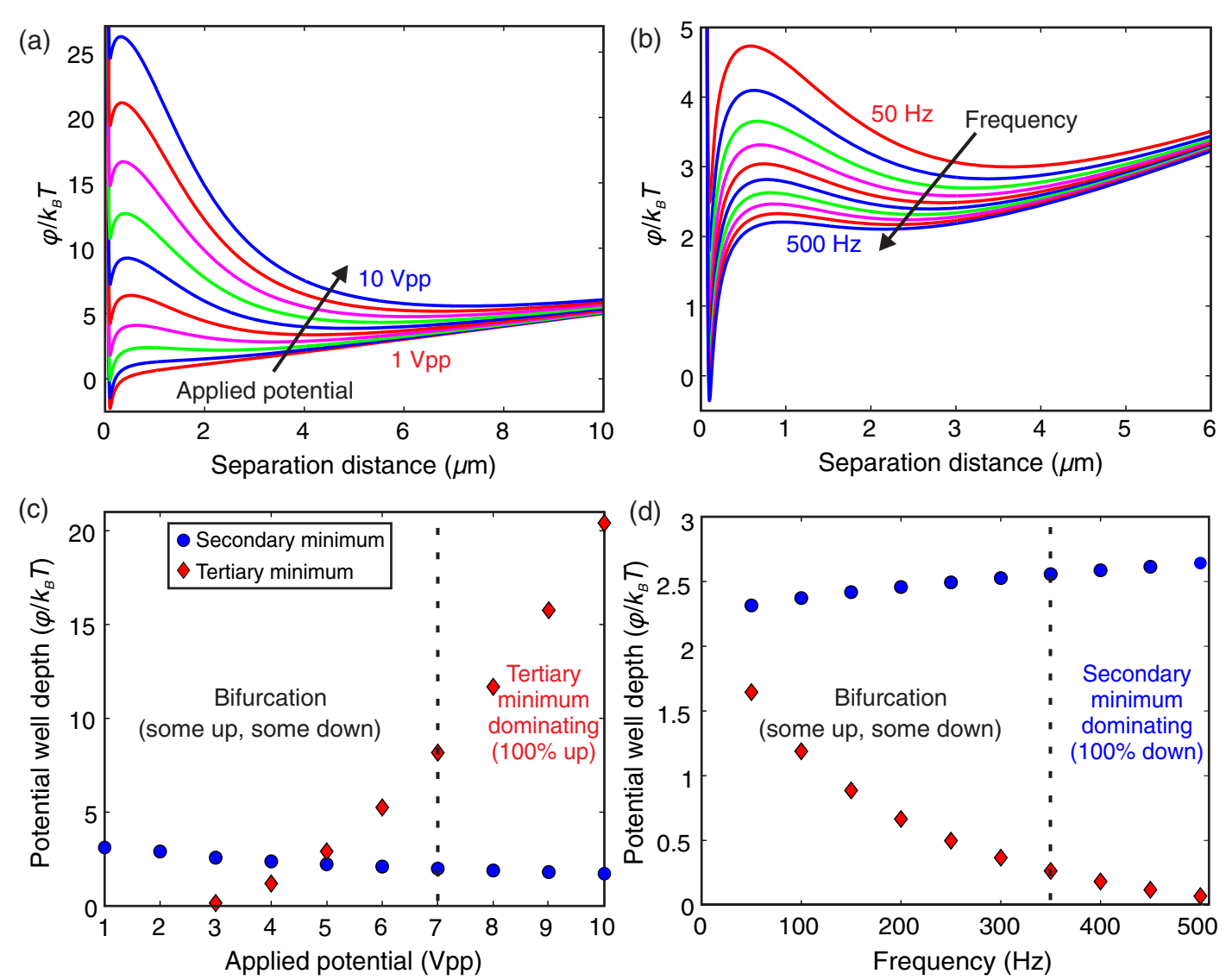

FIG. 6. Electric field dependence of the particle-electrode interaction potential for particles suspended in $1 \mathrm{mM} \mathrm{NaOH}$, calculated using Eq. (1). (a) Interaction potential as a function of voltage at $100 \mathrm{~Hz}$ (1 Vpp voltage steps between subsequent potential curves). (b) Interaction potential as a function of frequency at $4 \mathrm{Vpp}(50 \mathrm{~Hz}$ frequency steps between subsequent potential curves). (c), (d) Potential well depths of the secondary and tertiary minima as a function of applied potential (c) and frequency (d). The well depth is measured from the inflection point between the two minima. The vertical dashed lines denote the experimentally observed regimes shown in Fig. 4.

coincide with the voltages and frequencies observed experimentally, the model is qualitatively consistent with the electric-field-dependent bifurcation behavior in Fig. 4 and provides physical insight showing that the balance between EHD flow and gravity dictates the depth of the tertiary minimum.

To further test the model, we perform similar calculations for particles suspended in $1 \mathrm{mM} \mathrm{NaCl}$, an electrolyte in which we have never observed any height bifurcation (see Fig. S4 in the Supplemental Material [32]). Briefly, no tertiary minimum occurs in the interaction pseudopotential at $4 \mathrm{Vpp}$ for any frequency, and only at much higher voltages $(>8 \mathrm{Vpp})$ and lower frequencies $(100 \mathrm{~Hz})$ does a shallow tertiary minimum $\left(<\sim 0.5 k_{B} T\right)$ emerge. The absence of a deep tertiary minimum for particles in $\mathrm{NaCl}$ is due, in terms of the model, to a reduced EHD flow magnitude relative to particles in $\mathrm{NaOH}$, which in turn stems from a decrease in the particle zeta potential magnitude and the imaginary dipole coefficient [cf. $C_{0}^{\prime \prime}$ in Eq. (2)]. Simply put, for particles in $\mathrm{NaOH}$, the EHD flow is stronger and pushes the particles away from the electrode with more force than in $\mathrm{NaCl}$, creating the tertiary minimum through a balance with the gravitational force.

This calculation, although consistent with the observed lack of height bifurcation in $\mathrm{NaCl}$, raises a key point. The EHD flow, which we invoke here as the force pushing the particles away from the electrode, has been previously invoked as the mechanism giving rise to aggregation of particles in electrolytes like $\mathrm{NaCl}[21,28,29]$. This begs the question, if there is a flow directed radially inward toward particles near the electrode, why do the down particles not aggregate? The short answer is that sometimes they do: at very low frequencies $(25 \mathrm{~Hz})$, for particles in $1 \mathrm{mM} \mathrm{NaOH}$, we observe a simultaneous height bifurcation and aggregation of the down particles into hexagonally close-packed aggregates (see Supplemental material Fig. S2 [32]). It is unclear at present why we sometimes see apparent repulsion among the down particles and sometimes aggregation; it is possible that aggregation is favored only when certain multibody configurations occur, or when electrochemical reactions alter the local solution chemistry and corresponding field. Although the underlying details remain unclear, 
the key point here is that the observations shown in Fig. S2 [32] clearly demonstrate that particles simultaneously experience a set of forces that (i) cause them to move to distinct vertical heights over the electrode and (ii) induce an attractive lateral interaction that can result in aggregation. The EHD flow mechanism is broadly consistent with both of these observations.

More detailed numerical calculations of the electrically generated flow around the particles are needed to quantitatively model the height bifurcation, but the scaling analysis presented here captures the behavior of both bifurcating and nonbifurcating particles. The particleelectrode interaction pseudopotential provides an intuitive interpretation for the existence of a tertiary minimum and serves as a starting point for more detailed numerical and analytical considerations of the underlying physics, such as the particle dipole fields, EHD flow fields, and possible influence of electrochemical reactions.

\section{CONCLUSIONS}

In summary, we observe a surprising bifurcation in colloidal particle height above an electrode in lowfrequency oscillatory electric fields. Direct height measurements using confocal microscopy reveal that the particles are bifurcated by several particle radii, where down particles are $500 \mathrm{~nm}$ away from the electrode and up particles are at a height of $6-7 \mu \mathrm{m}$. The bifurcation is robust, as it occurs in several different suspending electrolytes and for several types of colloidal particles. Repeated bifurcation trials reveal that the likelihood for a particle to move up during the height bifurcation is truly random as the experimental distribution is statistically indistinguishable from a binomial distribution, indicating that particle height bifurcation is not due to participation in a distinct subpopulation of particles with different properties. The fraction of particles that move up during the bifurcation depends on the particle type, electrolyte, and electric field strength and frequency; in general, the fraction of particles moving up increases with increasing applied voltage and decreasing frequency. A vertical force balance employing a scaling estimate for the lift force due to the EHD flow yields an interaction pseudopotential landscape with a secondary and tertiary minimum at separation distances qualitatively consistent with the direct height measurements. The scaling model is qualitatively consistent with the random nature of the height bifurcation and the observed electric field dependence. The scaling analysis likewise agrees with the absence of a height bifurcation for particles suspended in $\mathrm{NaCl}$.

The results presented here raise several questions as well as intriguing possibilities. First, we observe the height bifurcation only in electrolytes where particles are primarily observed to strongly separate laterally from one another. Although we have some preliminary evidence that electrolytes which heretofore have been considered "separating" electrolytes do indeed yield aggregation of the down particles, it is unclear why they sometimes separate and sometimes aggregate. It is also unclear why the fraction of particles levitating up reaches a plateau at intermediate field strengths for some electrolytes but not others (cf. Fig. 4).

Despite these unanswered questions, the results clearly demonstrate the existence of a tertiary minimum in the interaction pseudopotential at micron-scale distances. Since colloids are often used as models of atomic scale systems, the tertiary minimum reported here could serve as a model energy landscape that is both highly tunable and easily experimentally accessible because of the micron length scales. Likewise, under appropriate conditions, the particles can reach surprisingly large heights - a phenomenon that could be manipulated to control the positioning of particles in lab-on-a-chip applications. For example, one can imagine applying a field to levitate particles up into a different stream or channel to control which direction the particles travel. Likewise, the height bifurcation occurs here for particles that are all ostensibly identical. If different particles undergo height bifurcations at different rates or with differing propensities to levitate, then it might be possible to rapidly separate the particle types. The results presented here serve as a framework for addressing these more complicated issues.

\section{ACKNOWLEDGEMENTS}

C. S. D. was supported by National Science Foundation Division of Atmospheric and Geospace Science. We thank the California League of Food Processors for partial support of this research.

[1] J. Perrin, Atoms (Van Nostrand, New York, 1916), pp. 89-95.

[2] W. B. Russel, D. A. Saville, and W. R. Schowalter, Colloidal Dispersions, 1st ed. (Cambridge University Press, Cambridge, England, 1991).

[3] P. Sarkar and P.S. Nicholson, Electrophoretic Deposition (EPD): Mechanisms, Kinetics, and Applications to Ceramics, J. Am. Ceram. Soc. 79, 1987 (1996).

[4] B. Bharti, G. H. Findenegg, and O. D. Velev, Co-Assembly of Oppositely Charged Particles into Linear Cluster and Chains of Controllable Length, Sci. Rep. 2, 1004 (2012).

[5] O. D. Velev and K. H. Bhatt, On-Chip Micromanipulation and Assembly of Colloidal Particles by Electric Fields, Soft Matter 2, 738 (2006).

[6] M. Parthasarathy and D. J. Klingenberg, Electrorheology: Mechanisms and Models, Mater. Sci. Eng. R 17, 57 (1996).

[7] W. D. Ristenpart, I. A. Aksay, and D. A. Saville, Electrically Guided Assembly of Planar Superlattices in Binary Colloidal Suspensions, Phys. Rev. Lett. 90, 128303 (2003).

[8] M. Trau, D. A. Saville, and I. A. Aksay, Field-Induced Layering of Colloidal Crystals, Science 272, 706 (1996). 
[9] S. R. Yeh, M. Seul, and B. I. Shraiman, Assembly of Ordered Colloidal Aggregates by Electric-Field-Induced Fluid Flow, Nature (London) 386, 57 (1997).

[10] W. D. Ristenpart, I. A. Aksay, and D. A. Saville, Electrohydrodynamic Flow around a Colloidal Particle near an Electrode with an Oscillating Potential, J. Fluid Mech. 575, 83 (2007).

[11] T. M. Squires and M.Z. Bazant, Induced-Charge ElectroOsmosis, J. Fluid Mech. 509, 217 (2004).

[12] M. Z. Bazant and T. M. Squires, Induced-Charge Electrokinetic Phenomena: Theory and Microfluidic Applications, Phys. Rev. Lett. 92, 066101 (2004).

[13] J. A. Fagan, P. J. Sides, and D. C. Prieve, Evidence of Multiple Electrohydrodynamic Forces Acting on a Colloidal Particle near an Electrode due to an Alternating Current Electric Field, Langmuir 21, 1784 (2005).

[14] P. Richetti, J. Prost, and P. Barois, Two-Dimensional Aggregation and Crystallization of a Colloidal Suspension of Latex Spheres, J. Phys. (Paris), Lett. 45, 1137 (1984).

[15] M. Bohmer, In Situ Observation of 2-Dimensional Clustering during Electrophoretic Deposition, Langmuir 12, 5747 (1996).

[16] M. Trau, D. A. Saville, and I. A. Aksay, Assembly of Colloidal Crystals at Electrode Interfaces, Langmuir 13, 6375 (1997).

[17] P. J. Sides, Electrohydrodynamic Particle Aggregation on an Electrode Driven by an Alternating Electric Field Normal to It, Langmuir 17, 5791 (2001).

[18] T. Gong and D.W. M. Marr, Electrically Switchable Colloidal Ordering in Confined Geometries, Langmuir 17, 2301 (2001).

[19] T. Y. Gong, D. T. Wu, and D. W. M. Marr, Two-Dimensional Electrohydrodynamically Induced Colloidal Phases, Langmuir 18, 10064 (2002).

[20] J. Kim, J. L. Anderson, S. Garoff, and P. J. Sides, Effects of Zeta Potential and Electrolyte on Particle Interactions on an Electrode under ac Polarization, Langmuir 18, 5387 (2002).

[21] W. D. Ristenpart, I. A. Aksay, and D. A. Saville, Assembly of Colloidal Aggregates by Electrohydrodynamic Flow: Kinetic Experiments and Scaling Analysis, Phys. Rev. E 69, 021405 (2004).

[22] J. A. Fagan, P. J. Sides, and D. C. Prieve, Vertical Oscillatory Motion of a Single Colloidal Particle Adjacent to an Electrode in an ac Electric Field, Langmuir 22, 9846 (2006).
[23] J. D. Hoggard, P. J. Sides, and D. C. Prieve, ElectrolyteDependent Pairwise Particle Motion near Electrodes at Frequencies below $1 \mathrm{kHz}$, Langmuir 23, 6983 (2007).

[24] J. D. Hoggard, P. J. Sides, and D. C. Prieve, ElectrolyteDependent Multiparticle Motion near Electrodes in Oscillating Electric Fields, Langmuir 24, 2977 (2008).

[25] D. C. Prieve, P. J. Sides, and C. L. Wirth, 2-D Assembly of Colloidal Particles on a Planar Electrode, Curr. Opin. Colloid Interface Sci. 15, 160 (2010).

[26] C. L. Wirth, R. M. Rock, P. J. Sides, and D. C. Prieve, Single and Pairwise Motion of Particles near an Ideally Polarizable Electrode, Langmuir 27, 9781 (2011).

[27] C. L. Wirth, P. J. Sides, and D.C. Prieve, Electrolyte Dependence of Particle Motion near an Electrode during ac Polarization, Phys. Rev. E 87, 032302 (2013).

[28] C. S. Dutcher, T. J. Woehl, N.H. Talken, and W. D. Ristenpart, Hexatic-to-Disorder Transition in Colloidal Crystals Near Electrodes: Rapid Annealing of Polycrystalline Domains, Phys. Rev. Lett. 111, 128302 (2013).

[29] T. J. Woehl, K. L. Heatley, C. S. Dutcher, N. H. Talken, and W. D. Ristenpart, Electrolyte-Dependent Aggregation of Colloidal Particles near Electrodes in Oscillatory Electric Fields, Langmuir 30, 4887 (2014).

[30] J. A. Fagan, P. J. Sides, and P. C. Prieve, Vertical Oscillatory Motion of a Single Colloidal Particle Adjacent to an Electrode in an ac Electric Field, Langmuir 18, 7810 (2002).

[31] A. V. Delgado, F. Gonzalez-Caballero, R. J. Hunter, L. K. Koopal, and J. Lyklema, Measurement and Interpretation of Electrokinetic Phenomena, J. Colloid Interface Sci. 309, 194 (2007).

[32] See Supplemental Material at http://link.aps.org/ supplemental/10.1103/PhysRevX.5.011023 for details on the direct particle height measurements with confocal microscopy, evidence for aggregation of down particles, and the full tertiary minimum scaling analysis.

[33] J. A. Fagan, P. J. Sides, and D. C. Prieve, Vertical Motion of a Charged Colloidal Particle near an ac Polarized Electrode with a Nonuniform Potential Distribution: Theory and Experimental Evidence, Langmuir 20, 4823 (2004).

[34] A. Ramos, H. Morgan, N. G. Green, and A. Castellanos, $a c$ Electrokinetics: A Review of Forces in Microelectrode Structures, J. Phys. D 31, 2338 (1998). 